\title{
Scalable domain decomposition preconditioners for heterogeneous elliptic problems ${ }^{1}$
}

\author{
Pierre Jolivet $^{\mathrm{a}, \mathrm{b}, \mathrm{c}, *}$, Frédéric Hecht ${ }^{\mathrm{b}, \mathrm{c}}$, Frédéric Nataf ${ }^{\mathrm{b}, \mathrm{c}}$ and Christophe Prud'homme ${ }^{\mathrm{d}}$ \\ ${ }^{a}$ Laboratoire J. Kuntzmannn, Université J. Fourier, Grenoble Cedex 9, France \\ E-mail: jolivet@ann.jussieu.fr \\ ${ }^{\mathrm{b}}$ Laboratoire J.-L. Lions, Université P. et M. Curie, Paris, France \\ E-mails: \{hecht,nataf\}@ann.jussieu.fr \\ ${ }^{\mathrm{c}}$ INRIA, ALPINES research team, Rocquencourt, France \\ d IRMA, Université de Strasbourg, Strasbourg Cedex, France \\ E-mail: prudhomme@unistra.fr
}

\begin{abstract}
Domain decomposition methods are, alongside multigrid methods, one of the dominant paradigms in contemporary large-scale partial differential equation simulation. In this paper, a lightweight implementation of a theoretically and numerically scalable preconditioner is presented in the context of overlapping methods. The performance of this work is assessed by numerical simulations executed on thousands of cores, for solving various highly heterogeneous elliptic problems in both 2D and 3D with billions of degrees of freedom. Such problems arise in computational science and engineering, in solid and fluid mechanics.

While focusing on overlapping domain decomposition methods might seem too restrictive, it will be shown how this work can be applied to a variety of other methods, such as non-overlapping methods and abstract deflation based preconditioners. It is also presented how multilevel preconditioners can be used to avoid communication during an iterative process such as a Krylov method.
\end{abstract}

Keywords: Linear solvers, divide and conquer, scalability

\section{Introduction}

Discretizations of partial differential equations used to model physical phenomena typically lead to larger and larger systems that cannot be solved directly and require advanced preconditioning techniques to ensure a fast convergence of iterative methods. As the current trend in high performance computing evolve towards more and more concurrency, recent results using domain decomposition preconditioners [20] and multigrid methods $[3,31]$ clearly show why these are the methods of choice for achieving high-throughput finite element simulations. Both are examples of the divide and conquer paradigm. On the one hand, for scalar

\footnotetext{
${ }^{1}$ This paper received a nomination for the Best Paper Award at the SC2013 conference and is published here with permission of ACM.

${ }^{*}$ Corresponding author: Pierre Jolivet, Laboratoire J.-L. Lions, Université P. et M. Curie, Paris, France. E-mail: jolivet@ ann.jussieu.fr.
}

equations such as the simulation of flows through porous media, multigrid methods are often the preferred methods. One the other hand, domain decomposition methods are widely used in solid mechanics, in multiphysics or for code coupling. While these methods can offer high efficiency for solving PDE in parallel, special care must be taken when solving highly heterogeneous and complex systems for ensuring convergence rates independent of the number of computing nodes and heterogeneities involved in the simulation: as it will be shown in this paper with a simple comparison between two preconditioners, the difference in number of iterations can be an order of magnitude. The novelty of this paper is two-fold: first, a local reformulation of the one-level Schwarz method, quickly presented in [17], is extended to efficiently assemble a two-level preconditioner by avoiding the need of global operators. Second, the parallel distribution of a coarse grid operator is presented, it can for example be 
used to fuse communications. Altogether, large-scale experiments are performed for two different PDE, with various high-order finite elements, using highly varying coefficients on unstructured meshes, where simple methods are obsolete. There are two main families of domain decomposition algorithms: overlapping Schwarz methods and iterative substructuring methods. While this work is more focused towards the former, the key idea is the same for both methods: to obtain convergence rates which are independent of the number of subdomains, also referred to as substructures, an additional component to provide global transfer of numerical information across all subproblems is needed. In this work, the construction of a so-called coarse operator is presented. In Section 2, some important notions about domain decomposition methods are recalled, in Section 3, the construction of the coarse operator is presented. Numerical results are gathered in Section 3.4, where problems of up to 22 billions unknowns in 2D, and 2 billions unknowns in $3 \mathrm{D}$ are solved on more than $16 \mathrm{~K}$ threads.

\section{Domain decomposition preconditioners}

Let $\Omega \subset \mathbb{R}^{d}$ ( $d=2$ or 3 ) be a domain whose associated mesh can be partitioned into $N$ non-overlapping meshes $\left\{\mathcal{T}_{i}\right\}_{1 \leqslant i \leqslant N}$ using graph partitioners such as METIS [18] or SCOTCH [8]. Let $V$ be the finite element space spanned by the finite set of $n$ basis functions $\left\{\phi_{i}\right\}_{1 \leqslant i \leqslant n}$ defined on $\Omega$. Typical finite element discretizations of a symmetric, coercive bilinear form $a: V \times V \rightarrow \mathbb{R}$ yield the following system to solve:

$$
A x=b,
$$

where $\left(A_{i j}\right)_{1 \leqslant i, j \leqslant n}=a\left(\phi_{j}, \phi_{i}\right),\left(b_{i}\right)_{1 \leqslant i \leqslant n}=\left(f, \phi_{i}\right)$, $f$ being in the dual space $V^{*}$. Now let $\left\{V_{i}\right\}_{1 \leqslant i \leqslant N}$ be the local finite element spaces defined on the domains associated to each $\left\{\Omega_{i}\right\}_{1 \leqslant i \leqslant N}$ where $\Omega_{i}$ is the subdomain defined as the union of all mesh elements in $\mathcal{T}_{i}$, for all $1 \leqslant i \leqslant N$. If $\delta$ is a positive integer, the overlapping decomposition $\left\{\mathcal{T}_{i}^{\delta}\right\}_{1 \leqslant i \leqslant N}$ is defined recursively as follow: $\mathcal{T}_{i}^{\delta}$ is obtained by including all elements of $\mathcal{T}_{i}^{\delta-1}$ plus all adjacent elements of $\mathcal{T}_{i}^{\delta-1}$. For $\delta=0, \mathcal{T}_{i}^{\delta}=\mathcal{T}_{i}$. An example of such a construction is given Fig. 1, for $\delta=2$. Now consider the restrictions $\left\{R_{i}\right\}_{1 \leqslant i \leqslant N}$ from $V$ to $\left\{V_{i}^{\delta}\right\}_{1 \leqslant i \leqslant N}$, the local finite element spaces on $\left\{\Omega_{i}^{\delta}\right\}_{1 \leqslant i \leqslant N}$, and a local partition of unity $\left\{D_{i}\right\}_{1 \leqslant i \leqslant N}$ such that

$$
\sum_{j=1}^{N} R_{j}^{T} D_{j} R_{j}=I_{n \times n} .
$$

Algebraically speaking, if $\left\{n_{i}\right\}_{1 \leqslant i \leqslant N}$ denotes the number of degrees of freedom in each local finite element spaces, then $R_{i}$ is a boolean matrix of size $n_{i} \times n$, and $D_{i}$ is a diagonal matrix of size $n_{i} \times n_{i}$ for all $1 \leqslant i \leqslant N$. In our experiments, we use a pretty simple partition of unity, already used for example in [19]. Let $\widetilde{\chi}_{i}$ be a continuous piecewise linear function (hence mesh elements vertices and degrees of freedom coincide) defined on $\Omega_{i}^{\delta}$ as such:

$$
\widetilde{\chi}_{i}= \begin{cases}1, & \text { on all nodes of } \mathcal{T}_{i}^{0}, \\ 1-\frac{m}{\delta}, & \text { on all nodes of } \mathcal{T}_{i}^{m} \backslash \mathcal{T}_{i}^{m-1} \\ \forall m \in[1 ; \delta] .\end{cases}
$$

The local partition of unity is defined as:

$$
\chi_{i}=\frac{\tilde{\chi}_{i}}{\left.\sum_{j=1}^{N} \tilde{\chi}_{j}\right|_{V_{i}^{\delta} \cap V_{j}^{\delta}}},
$$

so that the support of the non-negative function $\chi_{i}$ is $V_{i}^{\delta}$ and

$$
\sum_{i=1}^{N} \chi_{i}=1
$$

Using a linear interpolant from the finite element space of continuous piecewise linear function from $\Omega_{i}^{\delta}$ to $V_{i}^{\delta}$ (which is typically of higher order), $D_{i}$ can be obtained from $\chi_{i}$ for all $1 \leqslant i \leqslant N$. For more complex partitions of unity, see for example [6]. Using the partition of unity, a common one-level preconditioner for system (1) introduced in [7] is:

$$
\mathcal{P}_{\mathrm{RAS}}^{-1}=\sum_{i=1}^{N} R_{i}^{T} D_{i}\left(R_{i} A R_{i}^{T}\right)^{-1} R_{i} .
$$

Equation (3) clearly shows the need of the globally assembled matrix, or to be more precise, the need of $N$ assembled submatrices (commonly referred to as "Dirichlet" matrices). These are typically hard to assemble directly and independently with classical finite element packages, in comparison with unassembled matrices (or "Neumann" matrices). Two approaches can be considered to build each "Dirichlet" matrix. 
(1) Build $A$, then extract each assembled subproblem. While this is the natural approach, it usually require some communications to build a parallel structure capable of handling distributed degrees of freedom, with ghost elements.

(2) Build the stiffness matrices $A_{i}^{\delta+1}$ yielded by the discretization of $a$ on $V_{i}^{\delta+1}$, then, remove the columns and rows associated to degrees of freedom lying on elements of $\mathcal{T}_{i}^{\delta+1} \backslash \mathcal{T}_{i}^{\delta}$. This yields $A_{i}:=R_{i} A R_{i}^{T}$, the global assembled matrix $A$ is never assembled.

The second approach does not require any additional parallel information or communication: there is no need for a global ordering, associated to a global partition of the degrees of freedom. Local matrices $A_{i}$ are symmetric positive definite, as $A$ is.

Usually, one subdomain is mapped to a single MPI process. For that reason, a given MPI process has a natural access to $u_{i}:=R_{i} u$ for any function $u \in V$. In all the following, we will assume that a finite element function in $V_{i}^{\delta}$ (resp. $V$ ) can be interpreted as vector of $\mathbb{R}^{n_{i}}$ (resp. $\mathbb{R}^{n}$ ) for all $1 \leqslant i \leqslant N$. In order to compute a global sparse matrix-vector product $A x$, one has to notice that, thanks to the partition of unity and the duplication of unknowns on the overlap, it can be proven that:

$$
R_{i} A R_{j}^{T} D_{j} x_{j}=R_{i} R_{j}^{T} R_{j} A R_{j}^{T} D_{j} x_{j}
$$

so that

$$
\begin{aligned}
(A x)_{i} & =R_{i} A x=R_{i} A \sum_{j=1}^{N} R_{j}^{T} D_{j} R_{j} x \\
& =R_{i} \sum_{j=1}^{N} R_{j}^{T} R_{j} A R_{j}^{T} D_{j} R_{j} x \\
& =\sum_{j=1}^{N} R_{i} R_{j}^{T} A_{j} D_{j} x_{j} .
\end{aligned}
$$

Applying $R_{i} R_{j}^{T}$ to a vector $x_{j}$ of $V_{j}^{\delta}$ for all $1 \leqslant$ $i \neq j \leqslant N$ is equivalent to restricting $x_{j}$ to the degrees of freedom in $V_{j}^{\delta}$ that are duplicated within $V_{i}^{\delta}$, sending the resulting vector to subdomain $i$, which then prolongates by 0 the received vector outside of the overlap $V_{j}^{\delta} \cap V_{i}^{\delta}$. In the following, $\mathcal{O}_{i}$ will be the set of neighboring subdomains to $i$, i.e. $\{j: j \neq i$, $\left.V_{j}^{\delta} \cap V_{i}^{\delta} \neq \emptyset\right\}$ and $\overline{\mathcal{O}_{i}}=\mathcal{O}_{i} \cup\{i\}$. There is no need to assemble each $R_{i}$, one only needs to know the action of $\left\{R_{i} R_{j}^{T}\right\}_{1 \leqslant i \leqslant N, j \in \mathcal{O}_{i}}$.
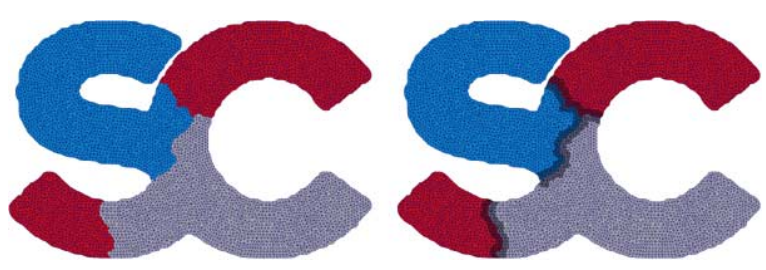

Fig. 1. Decomposition of the SC conference logo (no copyrights infringement intended) into three color-coded subdomains. On the left, the decomposition is non-overlapping, $\delta=0$. On the right, two consecutive extensions are performed, $\delta=2$, and represented in black overlay, to yield $\left\{\Omega_{i}^{2}\right\}_{1 \leqslant i \leqslant 3}$. (Colors are visible in the online version of the article; http://dx.doi.org/10.3233/SPR-140381.)

\subsection{Problem specification}

It is well known that one-level domain decomposition methods as depicted in the introduction of this section, see (3), do suffer from poor conditioning when used with many subdomains [26,29,33]. Indeed, if the subdomains are assumed to be of size $\mathcal{O}(H)$, then the condition number of the preconditioner grows as $1 / H$ for overlapping methods. For a given global mesh, increasing the number of subdomains leads to decreasing $H$, hence increasing the number of iterations needed for the preconditioned iterative method to converge. To overcome this recurrent problem in overlapping and non-overlapping methods, one must introduce a so-called coarse operator. In this work, an already established coarse operator whose theoretical foundations are presented in [30] is used. From a practical point of view, after building each local solver $A_{i}$, three dependent operators are needed:

(1) a deflation matrix $Z$ of size $n \times m$, with $m \ll n$,

(2) a coarse operator $E=Z^{T} A Z$ of size $m \times m$,

(3) the actual preconditioner

$$
\begin{aligned}
\mathcal{P}_{\mathrm{A}-\mathrm{DEF} 1}^{-1}= & \mathcal{P}_{\mathrm{RAS}}^{-1}\left(I-A Z E^{-1} Z^{T}\right) \\
& +Z E^{-1} Z^{T},
\end{aligned}
$$

thoroughly studied in [32].

The choice of using $\mathcal{P}_{\mathrm{A}-\mathrm{DEF} 1}^{-1}$ instead of, for example,

$$
\begin{aligned}
\mathcal{P}_{\mathrm{A}-\mathrm{DEF} 2}^{-1}= & \left(I-Z E^{-1} Z^{T} A\right) \mathcal{P}_{\mathrm{RAS}}^{-1} \\
& +Z E^{-1} Z^{T},
\end{aligned}
$$

which is also studied in [32] is pretty simple. While both preconditioners have similar numerical properties, applying $\mathcal{P}_{\mathrm{A}-\mathrm{DEF} 1}^{-1}$ to a vector $u$ requires only one 
coarse problem solution (used in two different operations afterwards):

$$
Z E^{-1} Z^{T} u
$$

On the other hand, applying $\mathcal{P}_{\mathrm{A}-\mathrm{DEF} 2}^{-1}$ requires two coarse problem solutions:

$$
Z E^{-1} Z^{T} u \text { and } Z E^{-1} Z^{T} \mathcal{P}_{\mathrm{RAS}}^{-1} u
$$

Because applying a coarse correction is the most communication-intensive operation when preconditioning an iterative method, as shown in Section 3.2, it is best to compute only one correction per iteration for scalability purposes.

In overlapping and non-overlapping decomposition methods, the deflation matrix is usually defined as:

$$
\begin{aligned}
Z & =\left[\begin{array}{llll}
R_{1}^{T} W_{1} & R_{2}^{T} W_{2} & \cdots & R_{N}^{T} W_{N}
\end{array}\right] \\
& \in \mathbb{R}^{n} \times \mathbb{R}^{\sum_{i=1}^{N} \nu_{i}} .
\end{aligned}
$$

The question is then, how to choose all those $W_{i}$, for all $1 \leqslant i \leqslant N$, to build a numerically scalable preconditioner? In [30], they are built as:

$$
\begin{aligned}
& \left\{W_{i}=\left[\begin{array}{llll}
D_{i} \Lambda_{i_{1}} & D_{i} \Lambda_{i_{2}} & \cdots & D_{i} \Lambda_{i_{\nu_{i}}}
\end{array}\right]\right. \\
& \left.\quad \in \mathbb{R}^{n_{i}} \times \mathbb{R}^{\nu_{i}}\right\}_{1 \leqslant i \leqslant N}
\end{aligned}
$$

A threshold criterion is used to select the $\nu_{i}$ eigenvectors $\left\{\Lambda_{i_{j}}\right\}_{1 \leqslant j \leqslant \nu_{i}}$ associated to the smallest eigenvalues in magnitude of the following local generalized eigenvalue problems:

$$
A_{i}^{\delta} \Lambda_{i}=\lambda_{i} D_{i} R_{i, 0}^{T} R_{i, 0} A_{i}^{\delta} D_{i} \Lambda_{i}
$$

where $A_{i}^{\delta}$ is the matrix yielded by the discretization of $a$ on $V_{i}^{\delta}$ (unassembled submatrix), and $R_{i, 0}$ is the restriction operator from $V_{i}^{\delta}$ to the overlap $V_{i}^{\delta} \cap\left(\bigcup_{j \in \mathcal{O}_{i}} V_{j}^{\delta}\right)$. If $\Omega_{i}^{\delta} \cap \partial \Omega=\emptyset, \Omega_{i}^{\delta}$ is commonly referred to as a floating subdomain. Hence, the bilinear form $a$ lacks essential boundary conditions on $V_{i}^{\delta}$, so that $A_{i}^{\delta}$ is now symmetric positive indefinite (compared to $A_{i}$, the assembled submatrix, which is always symmetric positive definite under the assumption that $A$ is). These independent and local eigenproblems are solved concurrently in order to find low eigenvalues that are in some sense close the lowest eigenvalues of the preconditioned system. It is well known that those are hurting the convergence rate of traditional Krylov methods, see $[9,23]$ for further details. With this construction of the coarse operator, it can be proven that the condition number of the preconditioned system is now independent of the size of the subdomains, of the number of subdomains, and of the heterogeneities in the physical coefficients. Once again, interested readers are referred to [30].

\section{Design of the coarse operator}

The goal of this section is to explain how a coarse operator built using deflation vectors can be efficiently assembled for large-scale simulations. This work is implemented in a light and versatile $\mathrm{C}++$ framework that is not directly linked to domain decomposition methods, meaning that it is possible to use it to assemble coarse operator with other abstract deflation vectors, for example as defined in [12] for simulations in cosmology. When applicable, the MPI calls and operations related to linear algebra (either from dense BLAS [5] or sparse BLAS such as Intel Math Kernel Library or cuSPARSE [24]) are provided in typewriter font.

\subsection{Assembling the coarse operator}

Looking at the general formulation of $E$ introduced in the previous section, two global sparse matrixmatrix products must be computed to assemble the coarse operator:

$$
A Z \text { then } Z^{T}(A Z) \text {. }
$$

However, it is possible to exploit the sparsity pattern of $Z$ to build $E$ more efficiently than with these two consecutive computations. By construction, the deflation matrix $Z$ is made of blocks of dense matrices of size $n_{i} \times \nu_{i}$ as displayed in Fig. 2. If there is more than one block for which there are non-zeros in a given row of $Z$, it means that one is dealing with a duplicated unknown (those which are in the overlap). From the simple example in Fig. 2 with 4 subdomains, one can infer that $\mathcal{O}_{1}=\{2\}, \mathcal{O}_{2}=\{1,3\}, \mathcal{O}_{3}=\{2,4\}$ and

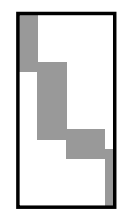

Fig. 2. Sparsity pattern of the deflation matrix $Z$ (grey blocks represent nonzero entries) with 4 subdomains. 
$\mathcal{O}_{4}=\{3\}$. Just like the globally assembled matrix $A$, the global deflation matrix $Z$ is never assembled, but its representation above is useful to understand the assembly of $E$. Instead, each subdomain has access to its local dense matrix $W_{i}$. The block $(i, j)$ of $E$ of size $\nu_{i} \times \nu_{j}$ is then equal to $W_{i}^{T} R_{i} A R_{j}^{T} W_{j}$. From that algebraic definition, one can see that the sparsity pattern of $E$ is linked to the connectivity between subdomains, because:

$$
R_{i} A R_{j}^{T}=0_{n_{i} \times n_{j}} \quad \Longleftrightarrow \quad V_{i}^{\delta} \cap V_{j}^{\delta}=\emptyset .
$$

In the context of overlapping domain decomposition methods, using Eqs (4)-(8), the block $(i, j)$ of $E$ can be computed as

$$
\begin{aligned}
W_{i}^{T} R_{i} A R_{j}^{T} W_{j} & =W_{i}^{T} R_{i} R_{j}^{T} R_{j} A R_{j}^{T} W_{j} \\
& =W_{i} R_{i} R_{j}^{T} A_{j} W_{j}
\end{aligned}
$$

As in Eq. (5), the previous equation shows how it is possible to take advantage of the duplicated unknowns on the overlap to compute a global product with only local computations and peer-to-peer transfers: the assembled matrices $A$ and $Z$ are not needed. Noticing that $R_{i} R_{i}^{T}=I_{n_{i} \times n_{i}}$, the subdomain $i$ needs to perform three tasks so that all blocks of $E$ can be computed:

(1) compute locally $T_{i}=A_{i} W_{i}$ (csrmm) and $E_{i, i}=W_{i}^{T} T_{i}$ (gemm),

(2) send to each neighboring subdomain $j \in \mathcal{O}_{i}$, $S_{j}=R_{j} R_{i}^{T} T_{i}$, and receive from each neighboring subdomain $U_{j}=R_{i} R_{j}^{T} T_{j}$,

(3) compute locally $E_{i, j}=W_{i}^{T} U_{j}$ (gemm).

The cost, in terms of peer-to-peer messages, of step 2 is approximately the same as one global sparse matrixvector product. Using the same toy problem as in Fig. 2, the following figure is a representation of the sparsity pattern of $E$. Two colors are used to differentiate blocks that can be computed without any communication and those that cannot. The three tasks to assemble all blocks of $E$ can be customized to suit one's needs using $\mathrm{C}++$ polymorphism. For example, in the context of non-overlapping methods, the sparsity pattern of $E$ is typically more dense: a block $(i, j)$ of $E$ is not null if and only if $j \in \mathcal{O}_{j}$, but also if there exists $k \in \mathcal{O}_{j}$ such that $k \in \mathcal{O}_{i}$. This can be handled by our framework. Likewise, each local computation $T_{i}$ as introduced in the first step, can be modified to involve more complex operations and communication patterns.
Stopping at that point in the construction of $E$, solving systems involving the coarse operator would require either to factorize a relatively small matrix on a large number of MPI processes, or to call an iterative method with once again, a distribution of data that would lead to a too fine-grained granularity (blocks of rows of $E$ are typically of size $\nu_{i}$ ranging from 1 to 30 ). In both cases, the increased communication overhead would lead to bad performances when solving multiple systems involving the coarse operator. Another approach would consist in replicating $E$ over all subdomains, and then performing independent factorizations, but that is simply not feasible for large decompositions, with arbitrary numbers of deflation vectors. In the following subsections, a more efficient data distribution is explained. While it will imply more communications during the setup of the coarse operator, it will cover all possible issues stated in this paragraph, regarding communication overhead, memory consumption and fast computation of coarse solutions.

\subsubsection{Master-slave approach}

The idea is to use only a "small" group of processes that will be in charge of factorizing the coarse operator and that will afterwards be called for computing solutions of systems involving $E^{-1}$ using a distributed sparse direct solver. This is inspired by the famous master-slave approach. For the rest of the paper, the following notations will be thoroughly used (for the sake of completeness, we provide their type in our implementation):

- unsigned short P: the number of masters, chosen at runtime by the user,

- MPI_Comm masterComm: a communicator between all masters, set to MPI_COMM_NULL on slaves, on which will be instantiated the distributed solver,

- MPI_Comm splitComm: a communicator between a master and its slaves in which the rank of the master is always 0 , and the ranks of the slave follow the same order as in MPI_COMM_WORLD.

A representation of such communicators is given Fig. 4. Prior to factorizing $E$, the first step is to assemble it in a distributed matrix on the masters. Each master will be in charge of assembling all the values of its slaves. It is assumed that the format in which the distributed matrix is stored is a simple global CSR or global $\mathrm{COO}$ - the standard format for most linear solvers available nowadays, meaning that for each nonzero value, one must know the absolute row and column indices of the given value in the global matrix $E$. 
For a process $i$, the global row indices $I_{E_{i}}$ of all the blocks $\left\{E_{i, j}\right\}_{j \in \overline{\mathcal{O}_{i}}}$ range from $r_{i}$ to $r_{i}+\nu_{i}$, where $r_{i}=\sum_{j=1}^{i-1} \nu_{j}$, and the global column indices $J_{E_{j}}$ of all the blocks $\left\{E_{j, i}\right\}_{j \in \overline{\mathcal{O}_{i}}}$ range from $r_{i}$ to $r_{i}+\nu_{i}$. The simplest approach would then be to:

(1) call MPI_Allgather $\left(\nu_{i}\right)$ on MPI_COMM WORLD to be able to compute the cumulative sums $\left\{r_{j}\right\}_{j \in \overline{\mathcal{O}_{i}}}$ and to allocate the buffers $S_{j}$ and $U_{j}$ for all $j \in \mathcal{O}_{i}$,

(2) assemble locally $\left\{E_{i, j}\right\}_{j \in \overline{\mathcal{O}_{i}}}$ as previously and store the values in CSR (or COO): $\left(I_{E_{i}}, J_{E_{i}}\right.$, $\left.\operatorname{val}_{E_{i}}\right)$,

(3) call MPI_Gatherv $\left(I_{E_{i}}\right), \quad$ MPI_ $\operatorname{Gatherv}\left(J_{E_{i}}\right)$, and MPI_Gatherv $\left(\operatorname{val}_{E_{i}}\right)$ on spli tComm with rank 0 as root.

The number of non-zero values for a process $i$ is:

$$
\begin{aligned}
\operatorname{size}\left(\operatorname{val}_{E_{i}}\right)= & \underbrace{\nu_{i} \times \nu_{i}}_{\text {Diagonal values in Fig. } 3} \\
& +\underbrace{\nu_{i} \times \sum_{j \in \mathcal{O}_{i}} \nu_{j}}_{\text {Off-diagonal values in Fig. } 3}
\end{aligned}
$$

meaning that prior to the three MPI_Gatherv in step 3, a call to MPI_Gatherv $\left(\mathcal{O}_{i}\right)$ on splitComm with rank 0 as root must be made to allocate the right buffers for the distributed format $I_{E}, J_{E}$ and $\mathrm{val}_{E}$ on each master. While this approach is somehow natural for assembling the distributed matrix on the masters because of the ordering of the rank of the slaves, calling MPI_Gatherv is similar to concatenating all local chunks of $E$ - it implies a lot of (unnecessary) communications. In particular, why should slaves send to masters the global row and column indices? Indeed, at the end of assembly, only the masters have access to the distributed coarse operator, so it is their responsibil-

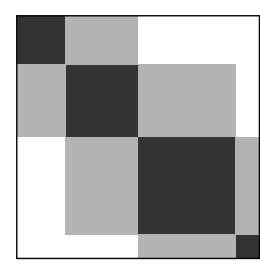

Fig. 3. Sparsity pattern of E. Diagonal blocks involve only local computations (step 1) while off-diagonal blocks also involve peerto-peer transfers (steps 2 and 3). ity to compute the indices. The slaves should not have to store or compute anything related to the distributed format. The following approach has the advantage of transferring only what is needed from one slave $i$ to its master: the array of scalar $\operatorname{val}_{E_{i}}$. The indices will be computed after reception by each master, meaning that the memory overhead on the slaves is null (no integers are allocated for storing any index). The new workflow is now:

(1) perform a neighborhood collective operation MPI_Ineighbor_alltoall $\left(\nu_{i}\right)^{2}$ on the communicator to which the distributed graph topology information of the connectivity between subdomains is attached (MPI_Dist_ graph_create_adjacent). Then allocate accordingly the buffers $S_{j}$ and $U_{j}$ for all $j \in \mathcal{O}_{i}$

(2) call MPI_Gather $\left(\left[\nu_{i},\left|\mathcal{O}_{i}\right|\right]\right)$ (array of 2 integers) on splitComm with rank 0 as root so that masters can preallocate the distributed CSR $\left(I_{E}, J_{E}, \operatorname{val}_{E}\right)$,

(3) assemble locally $\left\{E_{i, j}\right\}_{j \in \overline{\mathcal{O}_{i}}}$ as previously and send the values to the master. Prepend to the beginning of the message, the values of $\mathcal{O}_{i}$, i.e. the final size of the message is $\left|\mathcal{O}_{i}\right|+(11)$.

Additionally, the masters must concatenate all $\nu_{i}$ gathered in step 2, using MPI_Allgatherv on commMaster to be able to compute all cumulative sums $r_{i}$, for all $i \in \operatorname{splitComm.~This~call~is~equivalent~to~}$ the MPI_Allgather in step 1 of the "natural" algorithm, but this time it does not involve any slave. When a master receives a message from a slave $i$, it knows that the global row index ranges from $r_{i}$ to $r_{i}+\nu_{i}$, and because the first values of the received message are a copy of $\mathcal{O}_{i}$, it can compute the correct global column indices for the neighbors of this slave. The complete algorithm for assembling the coarse operator is summarized in Algorithms 1 (construction of all blocks of $E$ ) and 2 (distributed assembly on the masters).

\subsubsection{Electing the masters}

In the previous paragraph, the masters are defined in a rather abstract way, as the processes that have a rank equal to 0 in splitComm. We have two ways to define the aforementioned MPI communicator. The first is the natural distribution: the process are spread uniformly and contiguously into $P$ groups, the masters are of rank $i \cdot N / P$, for all $0 \leqslant i \leqslant P-1$. The second distribution is a little more advanced and better suited for assembling symmetric coarse operators. In

\footnotetext{
${ }^{2}$ New to the MPI-3 standard.
} 


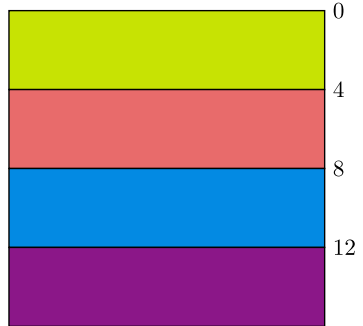

Uniform distribution
Non-uniform distribution

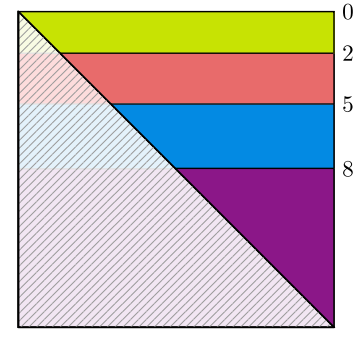

Fig. 4. Distribution of $E$ when built with 16 subdomains using 4 masters. Each color represents a different spli tComm, each number represents the rank of the master (in MPI_COMM_WORLD) of a given splitComm. On the right, the number of values per splitComm is roughly the same if the values below the diagonal are dropped (symmetric coarse operator). (Colors are visible in the online version of the article; http://dx.doi.org/10.3233/SPR-140381.)

that case, one only needs to assemble the upper part of the distributed CSR or COO, so that only the following blocks are computed and assembled:

$$
E_{i, j}, \quad \forall 1 \leqslant i \leqslant N, \forall j \in \overline{\mathcal{O}_{i}}: j \geqslant i .
$$

Moreover, only the upper parts of the dense diagonal blocks $E_{i, i}$ for all $1 \leqslant i \leqslant N$ are needed. To ensure load balancing between masters, the processes are now spread contiguously but non-uniformly, with masters of rank $p_{i}$, where $p_{i}$ is defined by the following sequence to ensure heuristically that the number of values within each quadrilateral in Fig. 4 is the same:

$$
\begin{aligned}
p_{0} & =0, \\
p_{i} & =\left\lfloor N-\sqrt{\left(p_{i-1}-N\right)^{2}-N^{2} / P}+0.5\right\rfloor, \\
& 1 \leqslant i \leqslant P-1 .
\end{aligned}
$$

The procedure compute in Algorithm 1 is in charge of returning a dense array of values corresponding to the block of $E$ given in argument, while the procedure assemble in Algorithm 2 is in charge of computing the indices of the values of the block of $E$ given in argument and store them in the distributed matrix representation.

\subsection{Applying a coarse operator correction}

Once $E$ has been assembled, it is involved in the solution of the following problem for a given vector $u$ : $Z E^{-1} Z^{T} u$. It is assumed that the right-hand side $Z^{T} u$ and the solution $E^{-1} Z^{T} u$ of the coarse problem are kept distributed on masterComm at all time, as input and output of the distributed solver. The computation of the correction can obviously be broken down into three basic operations:

(1) compute $Z^{T} u=w \in \mathbb{R}^{\sum_{i=1}^{N} \nu_{i}}$. Once again, the structure of $Z$ makes it possible to compute this sparse matrix-vector product without any explicit global representation of $Z$. Indeed,

$$
Z^{T} u=\sum_{i=1}^{N} W_{i}^{T} R_{i} u
$$

This is evaluated by having all subdomains $i$ compute locally $w_{i}=W_{i}^{T} u_{i}$ (gemv), and calling MPI_Gather $(v)$ on each splitComm with rank 0 as root to assemble $w$ with all local contributions of size $\nu_{i}$, for all $i$ in spli tComm, on the masters.

(2) compute $E^{-1} w=y \in \mathbb{R}^{\sum_{i=1}^{N} \nu_{i}}$. This operation must be as fast and reliable as possible, since it is carried out by only few masters. The numerical factorization of $E$ as computed in the previous paragraph during the assembly phase is reused for each forward elimination and back substitution.

(3) compute $Z y=z \in \mathbb{R}^{\sum_{i=1}^{N} n_{i}}$. This is the exact dual of step 1 . This operation reads:

$$
Z y=\sum_{i=1}^{N} R_{i}^{T} W_{i} y_{i}
$$

First, a call to MPI_Scatter $(\mathrm{v})$ is made on each splitComm with rank 0 as root so that each subdomain $i$ can retrieve its $y_{i}$ of size $\nu_{i}$ from its master. Then they all compute $z_{i}=W_{i} y_{i}$ (gemv). Finally,

$$
(Z y)_{i}=\sum_{j=1}^{N} R_{i} R_{j}^{T} z_{j}
$$

is computed using the same communication procedure as for the sparse matrix-vector product, see (5).

Using this construction, it is clear that using a non-uniform criterion $\nu_{i}$ for each subdomain leads to using MPI communications with varying counts of data from each process (hence the $\mathrm{V}$ in parentheses). Because these communications scale as $\mathcal{O}(N)$, it is preferable to call prior to assembling the coarse operator MPI_Allreduce $\left(\nu_{i}, \mathrm{MPI}\right.$ MAX $)$. That way, it is 

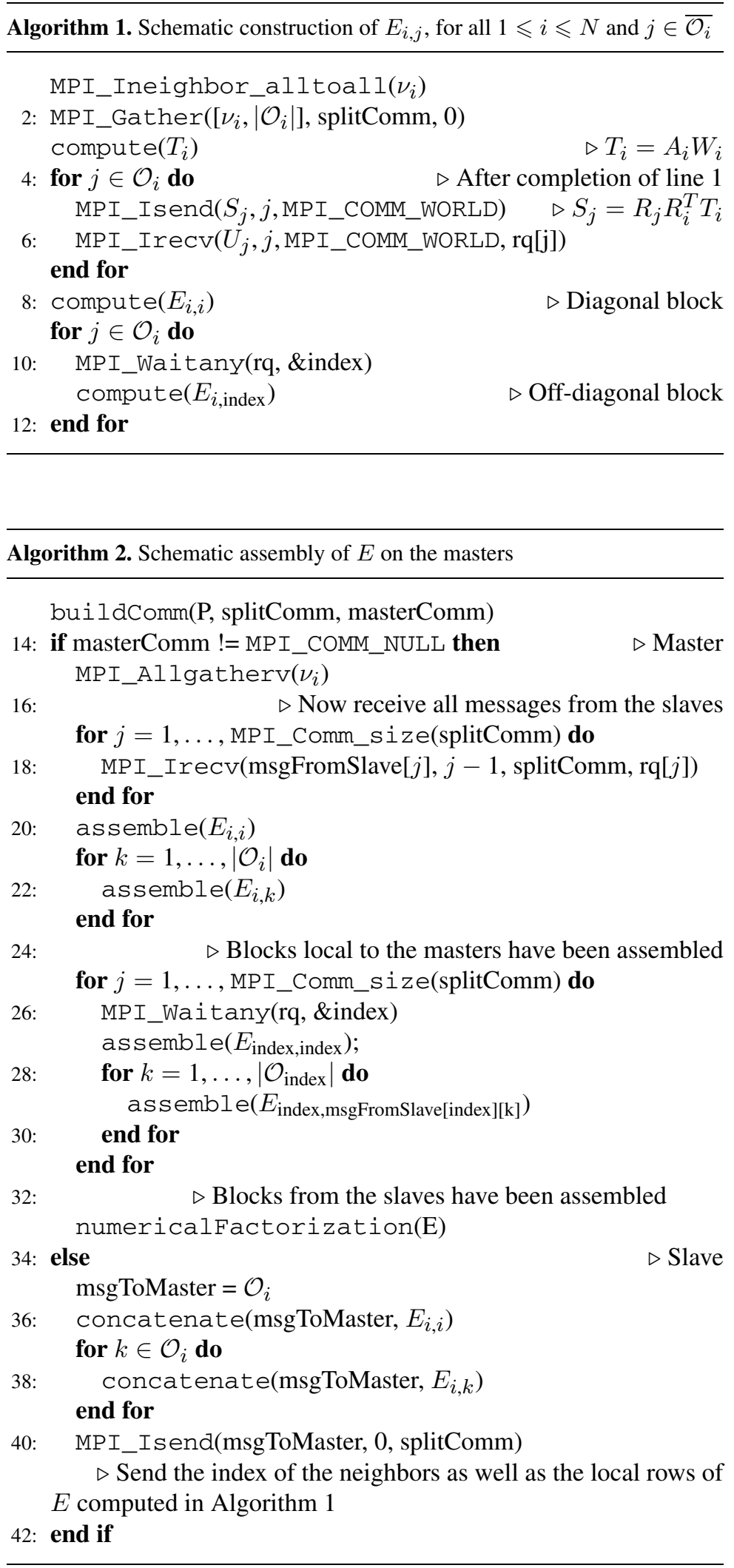
possible to use MPI communications with equal counts of data, which typically scale as $\mathcal{O}(\log (N))$. Moreover, the theoretical estimate on the condition number of the preconditioner system remains valid. There are also multiple simplifications possible during the assembly of $E$, because using a uniform criterion means that any process $i$ already knows the value of $\nu_{j}$, for all $j \in$ $\mathcal{O}_{i}$ without needing the call to MPI_Ineighbor_ alltoall and such.

\subsubsection{Fusing global reductions}

The workflow presented in the previous paragraph can be extended to provide a way to compute global reductions without making any call on MPI_COMM WORLD. Without loss of generality, let us assume the following in-place reduction is performed: MPI_ Allreduce ( $\alpha$, MPI_SUM, MPI_COMM_WORLD), where $\alpha$ is a scalar. Then,

(1) each process appends $\alpha$ to the send buffer $w_{i}$ defined in step 1 of Section 3.2,

(2) each master computes locally $\alpha=\mathrm{MPI}$ $\operatorname{SUM}_{i \in \operatorname{splitComm}}\left(\alpha_{i}\right)$ and performs an inplace reduction MPI_Allreduce $(\alpha$, MPI_SUM, masterComm).

(3) each master process appends $\alpha$ to the scattered vector $y$ defined in step 3 of Section 3.2 so that each slave can receive the globally reduced value.

Classical Krylov methods require global synchronizations, e.g. dot product, during the orthogonalization and the normalization of the basis vectors at each iteration, fusing those synchronizations inside, for example, a pipelined GMRES [11] yields an algorithm that does not require any global communication, yet data dependencies induced by the coarse solve introduce a "virtual" global synchronization.

\subsection{Cost analysis}

As a consequence of the observation made in the previous paragraph, it is assumed in this section that the number of deflation vectors per subdomain is chosen uniformly and set to $\nu$. This will also be the case in Section 3.4 for our scaling experiments, and we now propose a comprehensive summary of the cost of our two-level preconditioner, compared to a simple onelevel method. As a quick reminder, the cost of applying a one-level preconditioner such as $\mathcal{P}_{\text {RAS }}^{-1}$ in (3) is the same as computing $N$ independent solutions on each subdomain - application of $\left(R_{i} A R_{i}^{T}\right)^{-1}$, then performing one global matrix-vector product.

Memory footprint. Because it is clear that no global structure is needed for the construction and the use of
$Z$ and $E$ in our implementation, the memory footprint is straightforward to evaluate. For slaves, a dense array of size $\nu \times n_{i}$ is needed to store $W_{i}$. For masters, the same array must be stored, plus the factors of $E^{-1}$ (whose size depends on the distributed solver used), quantitative results are presented in Fig. 1 where the amount of memory needed to store the factors of $E^{-1}$ is displayed.

Messages. During the construction of $E$, each process $i$ must send and receive a single message from each neighboring subdomains $j \in \mathcal{O}_{i}$. The size of theses messages are

$$
\begin{gathered}
\nu \times \text { size of overlap between } \\
V_{i}^{\delta} \text { and } V_{j}^{\delta}
\end{gathered}
$$

see $S_{j}$ and $U_{j}$ in Algorithm 1. Then each slave $i$ must send a single message of double, of size $\left|\mathcal{O}_{i}\right|+$ $\left|\overline{\mathcal{O}_{i}}\right| \times \nu \times \nu$ to its master, see msgToMaster in Algorithm 2, and each master must reciprocally receive these message for all slaves in splitComm. During the solution, at each iteration, a MPI_Gather and a MPI_Scatter are called on each splitComm. The messages passed at the end of the correction step in Eq. (12) are of the exact same sizes as for the global matrix-vector product for the one-level method, cf. (5).

Computational intensity. The major contribution is the solution of each local and independent eigenvalue problem. Afterwards, for the setup of $E$, the BLAS computations stated in Section 3.1 are negligible with respect to the time spent transferring messages, and at each iteration the costly operation in Section 3.2 is the solution of the coarse correction - application of $E^{-1}$ using a distributed direct solver. Note that for big subdomains, this cost is negligible compared to the one of computing each local solution for the one-level preconditioner $\mathcal{P}_{\text {RAS }}^{-1}$, cf. (3).

\subsection{Numerical results}

Results in this section were obtained on Curie, a Tier-0 system for PRACE ${ }^{3}$ composed of 5040 nodes made of 2 eight-core Intel Sandy Bridge processors clocked at $2.7 \mathrm{GHz}$. The interconnect is an InfiniBand QDR full fat tree and the MPI implementation used was BullxMPI version 1.1.16.5. Intel compilers and Math Kernel Library in their version 13.1.0.146 were

\footnotetext{
${ }^{3}$ Partnership for Advanced Computing in Europe.
} 
used for all binaries and shared libraries, and as the linear algebra backend for both dense and sparse computations in our framework. $L U$ or $L D L^{T}$ decomposition of the local problems are computed with either instances of MUMPS [1,2] or PaStiX [16] on MPI_COMM_SELF, or preferably using Intel MKL PARDISO, University of Lugano PARDISO [27,28], or WSMP $[13,14]$ which are by design more suited for multithreaded computations. $L U$ or $L D L^{T}$ decomposition of the coarse operator is computed using MUMPS, PaStiX, or PWSMP. Eigenvalue problems to compute the deflation vectors as defined in (9) are solved using ARPACK [21] (procedure dsaupd). Finite element matrices are obtained from FreeFem $++[15]$, but the design of our domain decomposition framework makes it simple to port to other Domain-Specific (Embedded) Language such as Feel ++ [25], FEniCS [22] or finite element libraries like deal.II [4], GetFem ++ and such. We display the speedup and efficiency in terms of number of MPI processes. In these experiments, each MPI process is assigned a single subdomain, and 2 OpenMP threads using the following bindings: --bind-to-socket --bycore. The GMRES is stopped when a relative $10^{-6}$ decrease of the residual is reached.

First, the system of linear elasticity with highly heterogeneous elastic moduli is solved with a minimal geometric overlap of one mesh element. Its variational formulation reads:

$$
\begin{aligned}
a(u, v)= & \int_{\Omega} \frac{E \nu}{(1+\nu)(1-2 \nu)} \nabla \cdot u \nabla \cdot v \\
& +\frac{E}{1+\nu} \varepsilon(u): \varepsilon(v) \\
& +\int_{\Omega} f \cdot v+\int_{\partial \Omega} g \cdot v,
\end{aligned}
$$

where

- Young's modulus $E$ and Poisson's ratio $\nu$ vary between two sets of values, $\left(E_{1}, \nu_{1}\right)=\left(2 \cdot 10^{11}\right.$, $0.25)$ and $\left(E_{2}, \nu_{2}\right)=\left(10^{7}, 0.45\right)$.

- $\varepsilon$ is the linearized strain tensor, $f$ are the body forces (in this case, only the gravity), and $g$ are the surface force (in this case, a vertical loading is imposed on some parts of the geometries).

Such an equation typically arises in computational solid mechanics, for modeling small deformations of bodies. For less compressible materials ( $\nu$ closer to $0.5)$, it is more natural to switch to non-overlapping preconditioners. In $2 \mathrm{D}$, we use piecewise cubic basis functions $(\sim 33 \mathrm{nnz}$ per row). The system is of con-

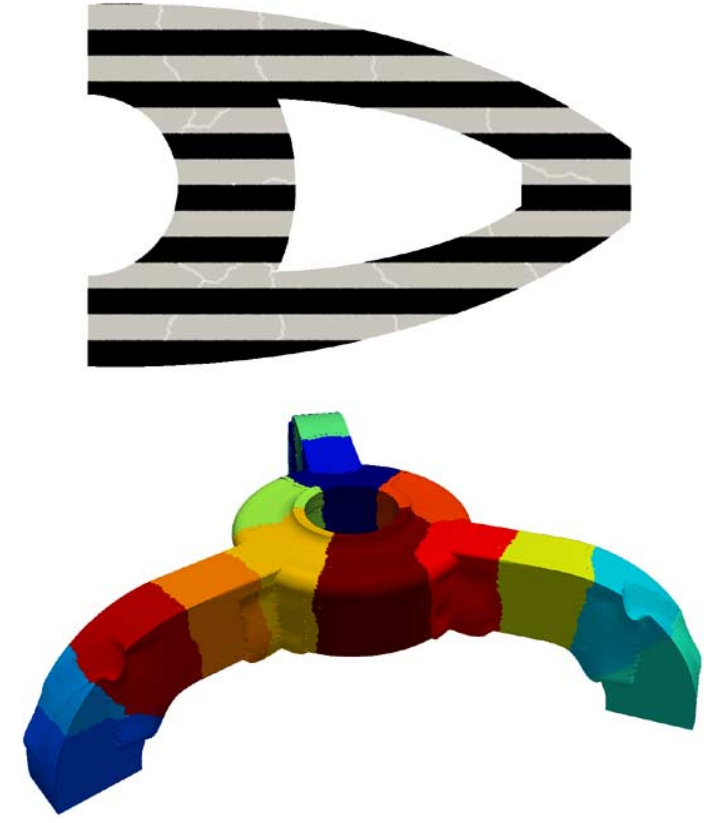

Fig. 5. Tripod used for our 3D and cantilever used for our 2D strong scaling experiments. Black and light grey are used to represent the variations of the Young's modulus (200 GPa and 0.01 GPa) and Poisson's ratio ( 0.25 and 0.45$)$. (Colors are visible in the online version of the article; http://dx.doi.org/10.3233/SPR-140381.)

stant size equal to approximately 2 billions unknowns. In $3 \mathrm{D}$, piecewise quadratic basis functions are used ( $~ 83 \mathrm{nnz}$ per row). The system is of constant size equal to approximately 300 million unknowns. Both geometries are displayed in Fig. 5 and were meshed by Gmsh [10] and partitioned with METIS.

After the partitioning step, each local mesh is refined concurrently by splitting each triangle or tetrahedron into multiple smaller elements. This means that we start the simulation with a relatively "coarse" global mesh (26 million triangles in 2D, 10 million tetrahedra in 3D), which is then refined in parallel (thrice in $2 \mathrm{D}$, twice in $3 \mathrm{D})$. We get a nice speedup from $1024 \times 2=2048$ to $8192 \times 2=16,384$ threads as shown in Fig. 7. According to the table in that figure, the costly operations in the construction of the preconditioner are the solution of each local eigenvalue problem (9) (column deflation), and the factorization of each local solver $A_{i}$ (column factorization). In $3 \mathrm{D}$, the complexity of such operations typically grows superlinearly with respect to the number of unknowns. That explains why we can achieve superlinear speedup. At peak performance, on 16,384 threads, the speedup relative to the runtime on 2048 threads equals $\frac{530.56}{51.76} \approx 10$. In $2 \mathrm{D}$, the computation costs are lower, and tend to scale better with the number of unknowns, 
which makes it harder to achieve high speedup for larger number of subdomains. At peak performance, on 16,384 threads, the speedup relative to the runtime on 2048 threads equals $\frac{213.20}{34.54} \approx 6$. In both cases, the solution of the eigenproblem is the limiting factor for achieving better speedups. This can be explained by the fact that the Arnoli method, which ARPACK is based on, tends to perform better for larger ratios $\frac{n_{i}}{\nu_{i}}$, but these values decrease as the subdomains get smaller. The number of deflation vectors per subdomain is constant and ranges from 20 to 15 . For larger but fewer subdomains, the time to compute the solution (column solution), i.e. the time for the GMRES to converge, is almost equal to the forward eliminations and back substitutions in the subdomains times the number of iterations. When the decompositions become bigger, subdomains are smaller, hence each local solution is computed faster and global communications have to be taken into account. To assess the need for such a sophisticated preconditioner, we display in Fig. 6 the convergence histogram of a simple one-level method versus this two-level method. One can easily understand that, while the cost of building the preconditioner cannot be neglected, it is necessary to ensure the convergence of the Krylov method: after more than $10 \mathrm{~min}$, the one-level barely decreases the relative error to $2 \cdot 10^{-5}$, while it takes $213.20 \mathrm{~s}$ for the two-level method, cf. Fig. 7 row \#5, to converge to the desired tolerance. That is at least a threefold speedup. Because one-level method are not numerically scalable, it is expected to get an even better speedup for larger decompositions.

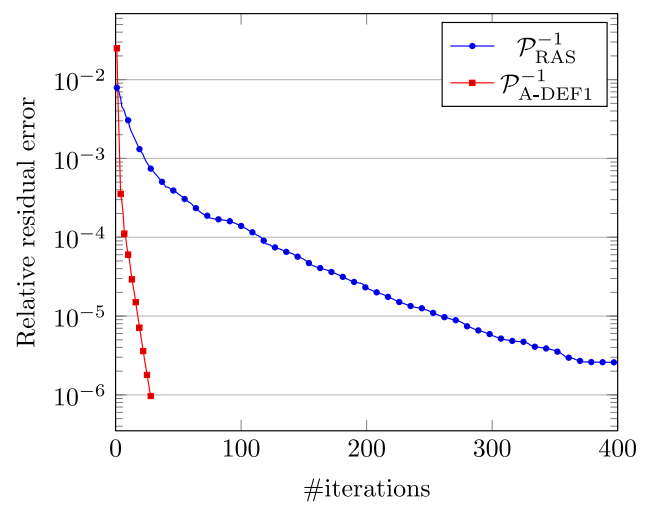

Fig. 6. Convergence of the GMRES(40) preconditioned by $\mathcal{P}_{\text {RAS }}^{-1}$ and $\mathcal{P}_{\mathrm{A}-\mathrm{DEF} 1}^{-1}$ for the problem of linear elasticity in $2 \mathrm{D}$ using 1024 subdomains. Timings for the setup and solution phases using $\mathcal{P}_{\mathrm{A}-\mathrm{DEF} 1}^{-1}$ are available in Fig. 7. Using $\mathcal{P}_{\mathrm{RAS}}^{-1}$, the convergence is not reached after $600 \mathrm{~s}$. (Colors are visible in the online version of the article; http://dx.doi.org/10.3233/SPR-140381.)
Moving on to the weak scaling properties of our framework, the problem we now solve is a scalar equation of diffusivity with highly heterogeneous coefficients (varying from 1 to $3 \cdot 10^{6}$ as displayed in Fig. 8) on $[0 ; 1]^{d}(d=2$ or 3$)$ with piecewise quartic basis functions in $2 \mathrm{D}(\sim 23 \mathrm{nnz}$ per row $)$, and piecewise quadratic basis functions in $3 \mathrm{D}(\sim 27 \mathrm{nnz}$ per row $)$. Its variational formulation reads:

$$
a(u, v)=\int_{\Omega} \kappa \nabla u \cdot \nabla v+\int_{\Omega} f \cdot v,
$$

where $f$ is a source term. Such an equation typically arises for modeling flows in porous media, or in computational fluid dynamics. No change is needed in our framework, since all operations are algebraic. The only work needed outside of our framework is changing the mesh used for computations, as well as the variational formulation of the problem in the FreeFem++ DSL.

On average, there is a constant number of degrees of freedom per subdomain equal to $280 \mathrm{~K}$ in $3 \mathrm{D}$, and near 2.7 millions in 2D. As for the strong scaling experiment, after building and partitioning a global "coarse" mesh (with few millions of elements), each local mesh is refined independently to ensure a constant size system per subdomain as the decomposition becomes bigger. The efficiency remains near the $90 \%$ mark, thanks to almost no variability in the factorization of the local problems and the construction of the deflation vectors. In 3D, the initial problem of 74 million unknowns is solved in $200 \mathrm{~s}$ on 512 threads. Using 16,384 threads, the problem is now made of approximately 2.3 billions unknowns, and it is solved in $215 \mathrm{~s}$, which yields an efficiency of $\sim 90 \%$. In $2 \mathrm{D}$, the initial problem of 695 million unknowns is solved in $175 \mathrm{~s}$ on 512 threads. Using 16,384 threads, the problem is now made of approximately 22.3 billions unknowns, and it is solved in $187 \mathrm{~s}$, which yields an efficiency of $\sim 96 \%$. At such scales, the most penalizing step in the algorithm is the construction of the coarse operator, specially in 3D, with a nonnegligible increase in the time spent to assemble $E$.

Finally, we present in this last paragraph the performances of our framework to assemble and factorize the coarse operator $E$ for all the previous simulations. Tables in Figs 7 and 9 already included these timings in their last column total $>$ factorization + deflation + solution), but for more in depth analysis, they are reported next separately.

Table 1 includes all timings relative to Algorithms 1 and 2 described in Section 3.1.1: the construction of the 


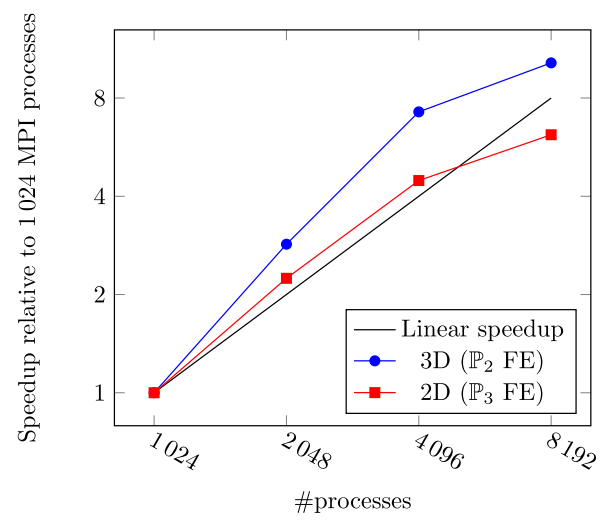

\begin{tabular}{|c|c|c|c|c|c|c|c|}
\hline & $N$ & Factorization (s) & Deflation (s) & Solution (s) & \#it. & Total (s) & \#d.o.f. \\
\hline \multirow[t]{4}{*}{$3 \mathrm{D}$} & 1024 & 177.86 & 264.03 & 77.41 & 28 & 530.56 & $293.98 \cdot 10^{6}$ \\
\hline & 2048 & 62.69 & 97.29 & 20.39 & 23 & 186.04 & \\
\hline & 4096 & 19.64 & 35.70 & 9.73 & 20 & 73.12 & \\
\hline & 8192 & 6.33 & 22.08 & 6.05 & 27 & 51.76 & \\
\hline \multirow[t]{4}{*}{$2 \mathrm{D}$} & 1024 & 37.01 & 131.76 & 34.29 & 28 & 213.20 & $2.14 \cdot 10^{9}$ \\
\hline & 2048 & 17.55 & 53.83 & 17.52 & 28 & 95.10 & \\
\hline & 4096 & 6.90 & 27.07 & 8.64 & 23 & 47.71 & \\
\hline & 8192 & 2.01 & 20.78 & 4.79 & 23 & 34.54 & \\
\hline
\end{tabular}

Fig. 7. Strong scaling experiments. (Colors are visible in the online version of the article; http://dx.doi.org/10.3233/SPR-140381.)

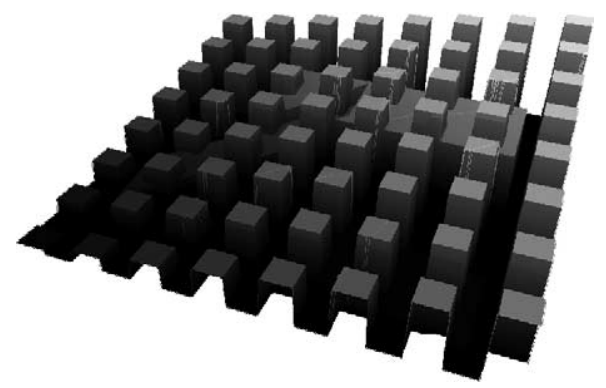

Fig. 8. Diffusivity $\kappa$ used for our 2D weak scaling experiment with channels and inclusions. Black and light grey are used to represent the variations of $\kappa$ from 1 to $3 \cdot 10^{6}$.

communicators, the assembly of $E$, and its numerical factorization. The constant number of deflation vectors computed per subdomain and assembled in the coarse operator is nothing else than $\frac{\operatorname{dim}(E)}{N}$. The most consuming part of the algorithm is the actual transfer and the assembly by the masters. Especially in 3D, when the coarse operator is becoming less and less sparse (directly linked with the average value of $\left|\mathcal{O}_{i}\right|$ ), it is likely to become a problem for even larger decomposition. Note that the MPI implementation used for these experiments is not thread compliant (and in particular, does not support a level of thread support equal to MPI_ THREAD_MULTIPLE), meaning that some "unnecessary" \#pragma omp critical had to be used during the assembly by the masters. At these scales, another problem is the factorization of $E$. Indeed, increasing the number of masters $P$ does not always have a beneficial effect for this concern, because distributed solvers have difficulties scaling beyond $\sim 128$ processes.

\section{Conclusion and outlook}

In this paper, we assess the efficiency of our implementation of a new adaptive two-level preconditioner suited for various problems such as linear elasticity or Darcy's law with high-contrast coefficients. The preconditioner was recently theoretically introduced in [30] and it provides a sound background for evaluating the performance of our portable framework. Using state of the art multithreaded direct linear solvers and eigensolvers and distributed direct linear solvers, we show experimentally that our approach is well suited for large-scale simulations in both 2D and 3D with high-order finite element methods, on up to 16,384 threads, enabling simulations for two classes of elliptic problems of more than 22 billion unknowns in $2 \mathrm{D}$ and 2 billion unknowns in 3D.

For building such a preconditioner, the deflation vectors used in our coarse operator are currently deter- 


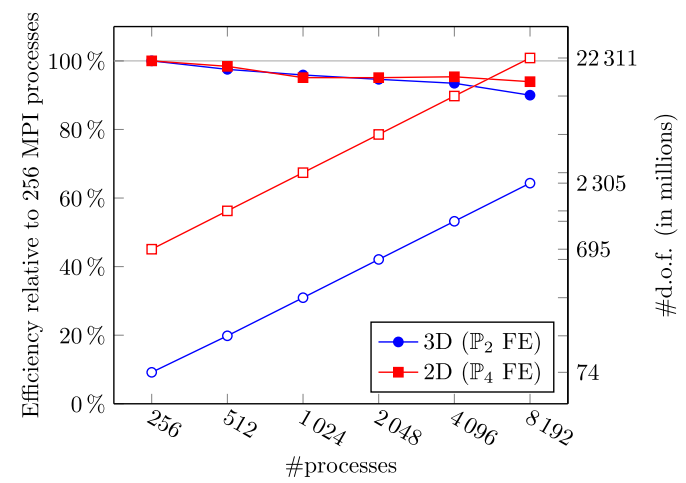

\begin{tabular}{|c|c|c|c|c|c|c|c|}
\hline & $N$ & Factorization (s) & Deflation (s) & Solution (s) & \#it. & Total (s) & \#d.o.f. \\
\hline \multirow[t]{6}{*}{$3 \mathrm{D}$} & 256 & 64.24 & 117.74 & 15.81 & 13 & 200.57 & $74.62 \cdot 10^{6}$ \\
\hline & 512 & 63.97 & 112.17 & 19.93 & 18 & 199.41 & $144.70 \cdot 10^{6}$ \\
\hline & 1024 & 63.22 & 118.58 & 16.18 & 14 & 202.40 & $288.80 \cdot 10^{6}$ \\
\hline & 2048 & 59.43 & 117.59 & 21.34 & 17 & 205.26 & $578.01 \cdot 10^{6}$ \\
\hline & 4096 & 58.14 & 110.68 & 27.89 & 20 & 207.47 & $1.15 \cdot 10^{9}$ \\
\hline & 8192 & 54.96 & 116.64 & 23.64 & 17 & 215.15 & $2.31 \cdot 10^{9}$ \\
\hline \multirow[t]{6}{*}{$2 \mathrm{D}$} & 256 & 29.40 & 111.35 & 25.71 & 29 & 175.85 & $695.96 \cdot 10^{6}$ \\
\hline & 512 & 29.60 & 111.52 & 27.99 & 28 & 179.07 & $1.39 \cdot 10^{9}$ \\
\hline & 1024 & 29.43 & 112.18 & 33.63 & 28 & 185.16 & $2.79 \cdot 10^{9}$ \\
\hline & 2048 & 29.18 & 112.23 & 33.74 & 28 & 185.20 & $5.58 \cdot 10^{9}$ \\
\hline & 4096 & 29.80 & 113.69 & 31.02 & 26 & 185.38 & $11.19 \cdot 10^{9}$ \\
\hline & 8192 & 29.83 & 113.81 & 30.67 & 25 & 187.57 & $22.31 \cdot 10^{9}$ \\
\hline
\end{tabular}

Fig. 9. Weak scaling experiments. (Colors are visible in the online version of the article; http://dx.doi.org/10.3233/SPR-140381.)

Table 1

Timings for assembling the coarse operator

\begin{tabular}{|c|c|c|c|c|c|c|c|c|c|c|c|}
\hline & $N$ & & & & & $\mid \mathcal{O}_{i}$ & age) & & $\begin{array}{l}\text { ost of } \\
\text { MB) }\end{array}$ & & \\
\hline $3 \mathrm{D}$ & 256 & 4 & & 5120 & & 11.5 & & 38 & & 2.78 & \\
\hline & 512 & 6 & & 10,240 & & 12.4 & & 78 & & 3.35 & \\
\hline & 1024 & 8 & 8 & 20,480 & 22,528 & 13.0 & 12.0 & 156 & 93 & 4.42 & 11.25 \\
\hline & 2048 & 12 & 12 & 40,960 & 40,960 & 13.8 & 12.9 & 332 & 138 & 6.91 & 5.68 \\
\hline & 4096 & 18 & 22 & 73,728 & 73,728 & 14.2 & 13.7 & 434 & 172 & 10.75 & 8.04 \\
\hline & 8192 & 64 & 48 & 131,072 & 131,072 & 14.7 & 14.6 & 420 & 241 & 19.92 & 17.30 \\
\hline $2 \mathrm{D}$ & 256 & 2 & & 5376 & & 5.5 & & 21 & & 9.39 & \\
\hline & 512 & 4 & & 10,240 & & 5.6 & & 32 & & 9.96 & \\
\hline & 1024 & 10 & 8 & 20,480 & 24,576 & 5.7 & 5.5 & 65 & 57 & 9.92 & 10.14 \\
\hline & 2048 & 14 & 12 & 38,912 & 40,960 & 5.8 & 5.7 & 94 & 83 & 10.05 & 6.20 \\
\hline & 4096 & 22 & 18 & 81,920 & 73,728 & 5.9 & 5.8 & 99 & 73 & 10.87 & 5.10 \\
\hline & 8192 & 36 & 36 & 163,840 & 122,880 & 5.9 & 5.8 & 152 & 118 & 13.27 & 6.96 \\
\hline
\end{tabular}

Note: Results are gathered two-by-two, the first column is for the diffusivity problem, the second is for the elasticity problem.

mined a priori by solving independently local eigenvalue problems. It is also possible to retrieve them a posteriori during the convergence of the iterative method, using for example approximations of the Ritz vectors. Using new theoretical results, we are currently evaluating a new a posteriori construction that does not require the solution of the aforementioned eigenvalue problems. This will hopefully improve our results for more fine-grained granularity in our strong scaling experiments. Finally, we are currently investing other types of systems for which domain decomposition methods have been proven to be efficient, namely 
nonlinear problems in computational solid mechanics, using non-overlapping methods. Thanks to the versatile design of our framework, and the flexibility of current finite element Domain-Specific (Embedded) Languages such as FreeFem ++ , new experiments should be soon possible.

\section{Acknowledgements}

This work has been supported in part by ANR through COSINUS program (project PETALh no. ANR-10-COSI-0013 and project HAMM no. ANR10-COSI-0009). It was granted access to the HPC resources of TGCC@CEA made available within the Distributed European Computing Initiative by the PRACE-2IP, receiving funding from the European Community's Seventh Framework Programme (FP7/2007-2013) under grant agreement RI-283493. The first author would like to thank A. Gupta and O. Schenk for providing him suitable licenses of their respective software (WSMP and PARDISO).

\section{References}

[1] P. Amestoy, I. Duff, J.-Y. L'Excellent and J. Koster, A fully asynchronous multifrontal solver using distributed dynamic scheduling, SIAM Journal on Matrix Analysis and Applications 23(1) (2001), 15-41.

[2] P. Amestoy, A. Guermouche, J.-Y. L'Excellent and S. Pralet, Hybrid scheduling for the parallel solution of linear systems, Parallel Computing 32(2) (2006), 136-156.

[3] A. Baker, R. Falgout, T. Kolev and U. Yang, Scaling hypre's multigrid solvers to 100,000 cores, in: High-Performance Scientific Computing, Springer, 2012, pp. 261-279.

[4] W. Bangerth, R. Hartmann and G. Kanschat, deal.II a general-purpose object-oriented finite element library, $A C M$ Transactions on Mathematical Software 33(4) (2007), 24-27.

[5] L. Blackford, A. Petitet, R. Pozo, K. Remington, R. Whaley, J. Demmel, J. Dongarra, I. Duff, S. Hammarling, G. Henry et al., An updated set of basic linear algebra subprograms (BLAS), ACM Transactions on Mathematical Software 28(2) (2002), 135-151.

[6] X.-C. Cai, M. Dryja and M. Sarkis, Restricted additive Schwarz preconditioners with harmonic overlap for symmetric positive definite linear systems, SIAM Journal on Numerical Analysis 41(4) (2003), 1209-1231.

[7] X.-C. Cai and M. Sarkis, Restricted additive Schwarz preconditioner for general sparse linear systems, SIAM Journal on Scientific Computing 21(2) (1999), 792-797.

[8] C. Chevalier and F. Pellegrini, PT-Scotch: a tool for efficient parallel graph ordering, Parallel Computing 34(6) (2008), 318-331.

[9] J. Frank and C. Vuik, On the construction of deflation-based preconditioners, SIAM Journal on Scientific Computing 23(2) (2002), 442-462.
[10] C. Geuzaine and J.-F. Remacle, Gmsh: A 3-d finite element mesh generator with built-in pre- and post-processing facilities, International Journal for Numerical Methods in Engineering 79(11) (2009), 1309-1331.

[11] P. Ghysels, T. Ashby, K. Meerbergen and W. Vanroose, Hiding global communication latency in the GMRES algorithm on massively parallel machines, SIAM Journal on Scientific Computing 1(35) (2013), 48-71.

[12] L. Grigori, R. Stompor and M. Szydlarski, A parallel twolevel preconditioner for cosmic microwave background mapmaking, in: Proceedings of the 2012 ACM/IEEE Conference on Supercomputing, SC12, IEEE Computer Society, 2012.

[13] A. Gupta, WSMP: Watson sparse matrix package - Part II: Direct solution of general systems, Technical Report 21888, IBM T.J. Watson Research Center, 2000.

[14] A. Gupta and H. Avron, WSMP: Watson sparse matrix package - Part I: Direct solution of symmetric systems, Technical Report 21886, IBM T.J. Watson Research Center, 2000.

[15] F. Hecht, S. Auliac, O. Pironneau, J. Morice, A. Le Hyaric and K. Ohtsuka, FreeFem++, available at: http://www.freefem.org/ ff++/.

[16] P. Hénon, P. Ramet and J. Roman, PaStiX: a high performance parallel direct solver for sparse symmetric positive definite systems, Parallel Computing 28(2) (2002), 301-321.

[17] P. Jolivet, V. Dolean, F. Hecht, F. Nataf, C. Prud'homme and N. Spillane, High performance domain decomposition methods on massively parallel architectures with FreeFem ++ , Journal of Numerical Mathematics 20(4) (2012), 287-302.

[18] G. Karypis and V. Kumar, A fast and high quality multilevel scheme for partitioning irregular graphs, SIAM Journal on Scientific Computing 20(1) (1998), 359-392.

[19] J.-H. Kimn and M. Sarkis, Restricted overlapping balancing domain decomposition methods and restricted coarse problems for the Helmholtz problem, Computer Methods in Applied Mechanics and Engineering 196(8) (2007), 1507-1514.

[20] A. Klawonn and O. Rheinbach, Highly scalable parallel domain decomposition methods with an application to biomechanics, ZAMM - Journal of Applied Mathematics and Mechanics/Zeitschrift für Angewandte Mathematik und Mechanik 90(1) (2010), 5-32.

[21] R. Lehoucq, D. Sorensen and C. Yang, ARPACK Users' Guide: Solution of Large-Scale Eigenvalue Problems with Implicitly Restarted Arnoldi Methods, Society for Industrial and Applied Mathematics, Vol. 6, 1998.

[22] A. Logg, K.-A. Mardal and G. Wells, Automated Solution of Differential Equations by the Finite Element Method, Vol. 84, Springer, 2012

[23] R. Nicolaides, Deflation of conjugate gradients with applications to boundary value problems, SIAM Journal on Numerical Analysis 24(2) (1987), 355-365.

[24] NVIDIA, CUDA Sparse Matrix library, available at: https://developer.nvidia.com/cusparse.

[25] C. Prud'homme, V. Chabannes, V. Doyeux, M. Ismail, A. Samake and G. Pena, Feel++: A computational framework for Galerkin methods and advanced numerical methods, in: Proceedings ESAIM, Vol. 38, 2012, pp. 429-455.

[26] A. Quarteroni and A. Valli, Domain Decomposition Methods for Partial Differential Equations, Vol. 10, Clarendon Press, 1999. 
[27] O. Schenk and K. Gärtner, Solving unsymmetric sparse systems of linear equations with PARDISO, Future Generation Computer Systems 20(3) (2004), 475-487.

[28] O. Schenk and K. Gärtner, On fast factorization pivoting methods for sparse symmetric indefinite systems, Electronic Transactions on Numerical Analysis 23 (2006), 158-179.

[29] B. Smith, P. Bjørstad and W. Gropp, Domain Decomposition: Parallel Multilevel Methods for Elliptic Partial Differential Equations, Cambridge Univ. Press, 2004.

[30] N. Spillane, V. Dolean, P. Hauret, F. Nataf, C. Pechstein and R. Scheichl, A robust two-level domain decomposition preconditioner for systems of PDEs, Comptes Rendus Mathematique 349(23) (2011), 1255-1259.
[31] H. Sundar, G. Biros, C. Burstedde, J. Rudi, O. Ghattas and G. Stadler, Parallel geometric-algebraic multigrid on unstructured forests of octrees, in: Proceedings of the 2012 ACM/IEEE Conference on Supercomputing, SC12, IEEE Computer Society, 2012.

[32] J. Tang, R. Nabben, C. Vuik and Y. Erlangga, Comparison of two-level preconditioners derived from deflation, domain decomposition and multigrid methods, Journal of Scientific Computing 39(3) (2009), 340-370.

[33] A. Toselli and O. Widlund, Domain Decomposition MethodsAlgorithms and Theory, Series in Computational Mathematics, Vol. 34, Springer, 2005. 

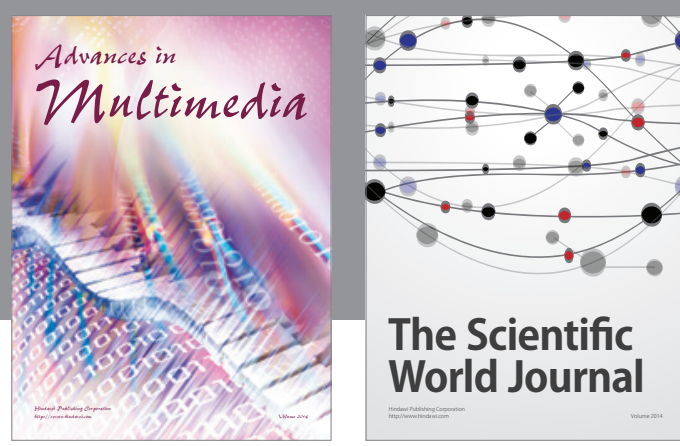

The Scientific World Journal
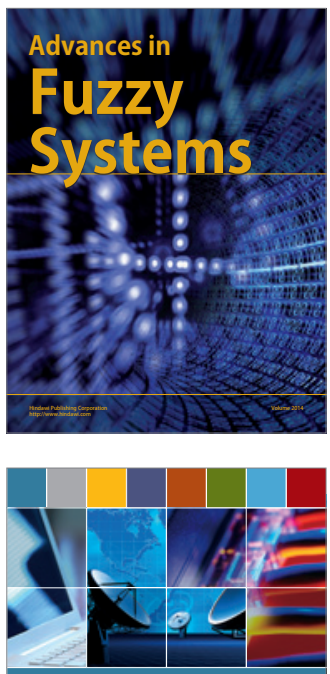

Computer Networks and Communications
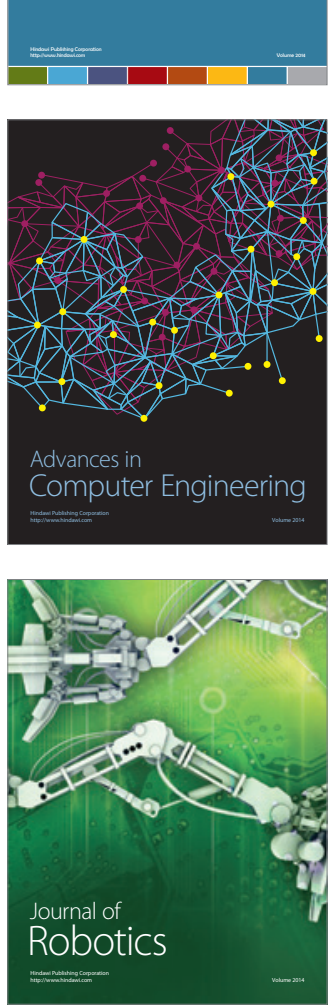
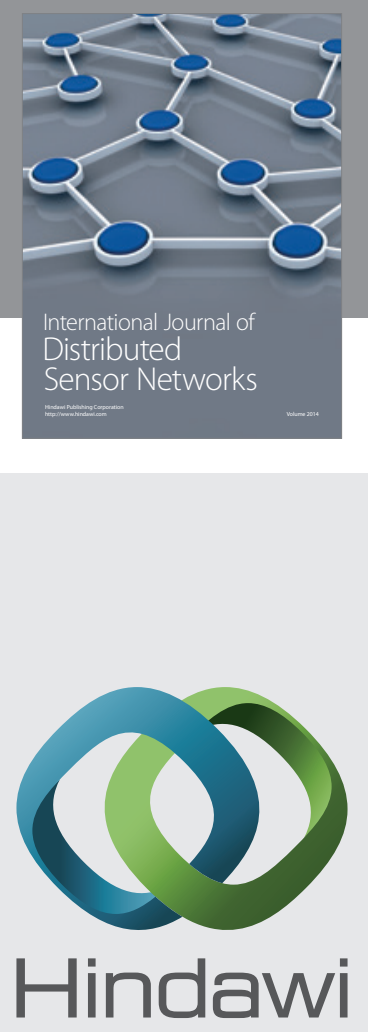

Submit your manuscripts at

http://www.hindawi.com
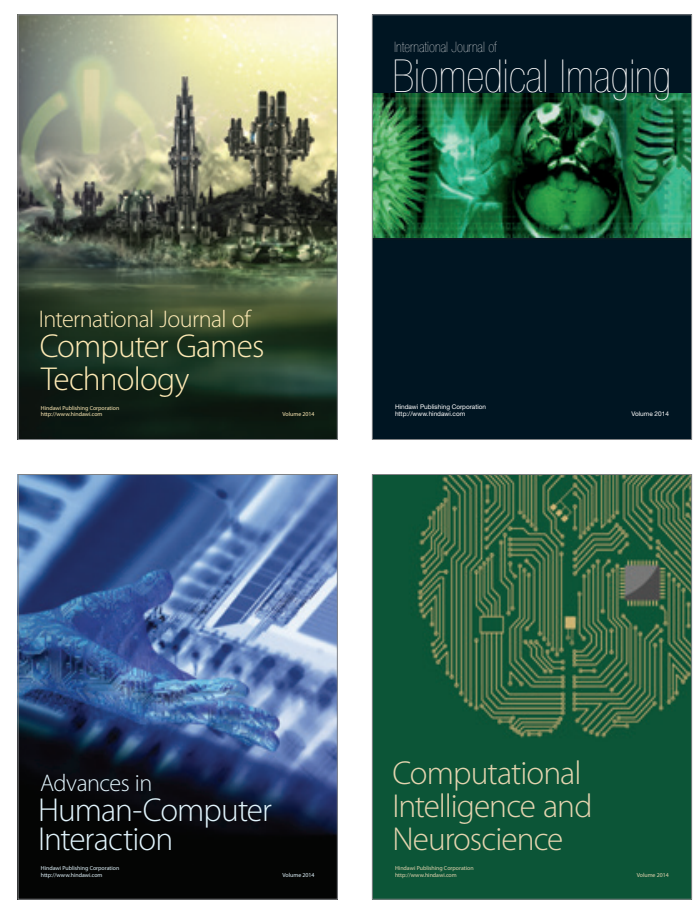
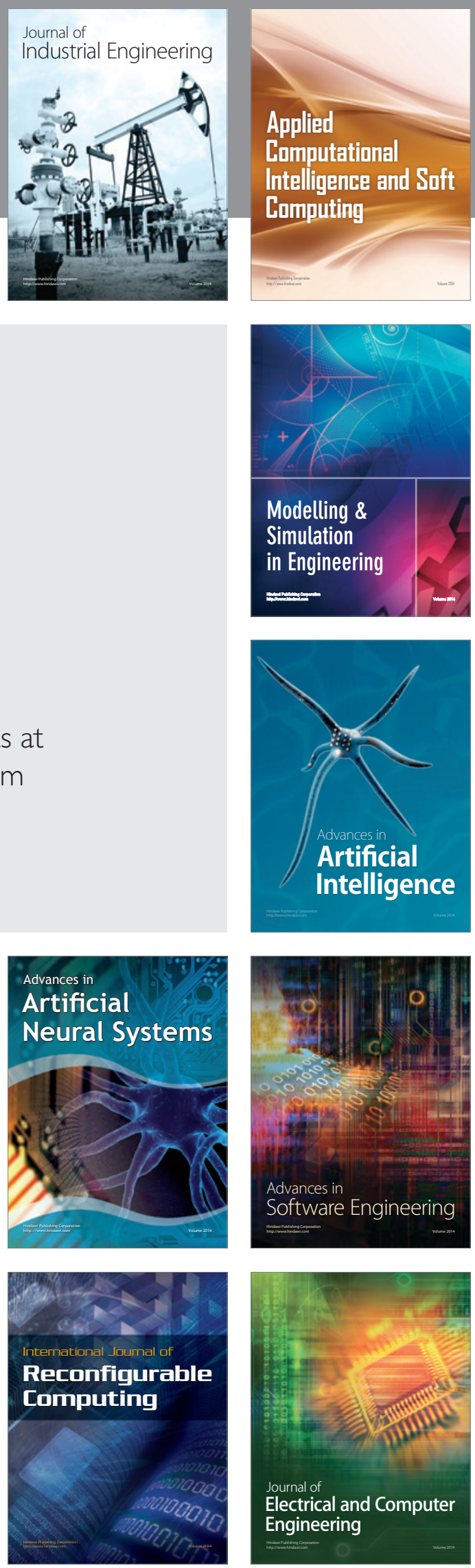